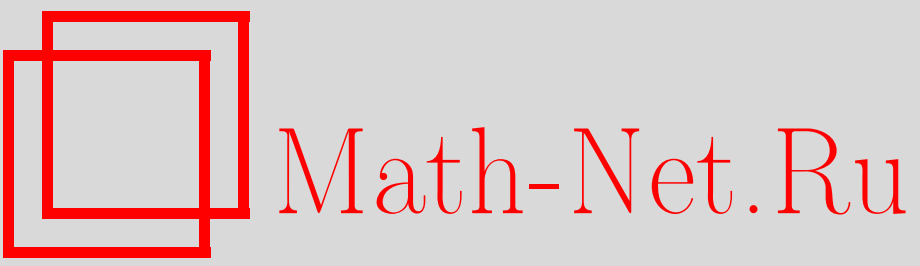

С. А. Довбыш, Оптимальные ляпуновские метрики гомеоморфизмов, обладающих гиперболической структурой, УМH, 2002, том 57, выпуск 4, 173-174

DOI: https://doi.org/10.4213/rm540

Использование Общероссийского математического портала Math-Net.Ru подразумевает, что вы прочитали и согласны с пользовательским соглашением

http://www.mathnet.ru/rus/agreement

Параметры загрузки:

IP: 54.197 .217 .227

26 апреля 2023 г., 04:26:51 


\title{
ОПТИМАЛЬНЫЕ ЛЯПУНОВСКИЕ МЕТРИКИ ГОМЕОМОРФИЗМОВ, ОБЛАДАЮЩИХ ГИПЕРБОЛИЧЕСКОЙ СТРУКТУРОЙ
}

\author{
С. А. ДовБыш
}

1. Напомним некоторые сведения [1], [2]. Пусть $f: X \rightarrow X$ - гомеоморфизм компактного метрического пространства $X$ с метрикой $\rho(\cdot, \cdot)$, локальные (радиуса $\delta$ ) устойчивое $W_{\delta}^{S}(x)$ и неустойчивое $W_{\delta}^{u}(x)$ "многообразия" точки $x \in X$ определяются как

$$
W_{\delta}^{S}(x)=\left\{y: \rho\left(f^{n} x, f^{n} y\right) \leqslant \delta, n \geqslant 0\right\}, \quad W_{\delta}^{u}(x)=\left\{y: \rho\left(f^{n} x, f^{n} y\right) \leqslant \delta, n \leqslant 0\right\} .
$$

ОПРЕдЕЛЕНИЕ 1. Гомеоморфизм $f$ обладает гиперболической структурой $(\Gamma \mathrm{C})$, а метрика $\rho$ называется гиперболической, если существуют такие $\delta>0, c \geqslant 1$ и $0<\lambda<1$, что

$$
y \in W_{\delta}^{s}(x)\left(y \in W_{\delta}^{u}(x)\right) \Longrightarrow \rho\left(f^{n} x, f^{n} y\right) \leqslant c \lambda^{|n|} \rho(x, y)
$$

при $n \geqslant 0(n \leqslant 0)$. Метрика назьвается ляпуновской, если при этом $c=1$.

Пусть $\Lambda_{s}$ и $\Lambda_{u}$ - точные нижние грани для констант $\lambda$ таких, что при подходящих $c \geqslant 1$ выполнены неравенства (1), относящиеся, соответственно, к устойчивым и неустойчивым многообразиям. Константы $\Lambda_{s}$ и $\Lambda_{u}$ не изменятся при выборе меньшего радиуса $\delta$ локальнх многообразий.

ОПРЕДЕЛЕНИЕ 2. Гомеоморфизм $f$ (вернее, пространство $X$ относительно гомеоморфизма $f$ ) обладает локальной структурой произведения (ЛСП), если существуют такие $\varepsilon>0$ и $\gamma>0$, что при $\rho(x, y) \leqslant \varepsilon$ пересечение $W_{\gamma}^{s}(x) \cap W_{\gamma}^{u}(y)$ состоит из единственной точки, обозначаемой $[x, y]$, и отображение $[\cdot, \cdot]:\{(x, y) \in X \times X: \rho(x, y) \leqslant \varepsilon\} \rightarrow X$ непрерывно.

Аксиома $\mathrm{A}^{\#}$ [1]-[3]. Гомеоморфизим $f$ на метрическом компакте $(X, \rho)$ удовлетворяет аксиоме $\mathrm{A}^{\#}$, или является $\mathrm{A}^{\#}$-гомеоморфизмом, если он одновременно обладает и ГС и ЛСП, а метрика $\rho$ является ляпуновской.

Аксиома А\# фактически появилась в [4] и была в явном виде сформулирована В. М. Алексеевым и М.В. Якобсоном.

Было доказано [5], что у гомеоморфизма $f$, обладающего ГС, имеется ляпуновская метрика, эквивалентная исходной (т.е. определяющая ту же топологию), причем эту метрику можно выбрать так [6], что оба отображения $f$ и $f^{-1}$ будут липшицевьми относительно нее. Кроме того, непрерывность отображения $[\cdot, \cdot \cdot]$ легко следует из его однозначности, см. [7]. Эти результаты фактически дают ответы на вопросы, поставленные в [1; с. 170], относительно возможности ослабления условий аксиомы $\mathrm{A}^{\#}$. (См. также обзор [8] эквивалентных определений гомеоморфизмов, обладающих ГС и ЛСП.) Далее, доказано [6], что у гомеоморфизма, обладающего ГС и ЛСП, имеется ляпуновская метрика (эквивалентная исходной), относительно которой этот гомеоморфизм является $\mathscr{L}$-гиперболическим в смысле следующего определения.

ОПредЕЛЕниЕ 3 [6]. Гомеоморфизм $f$ метрического компакта $\left(X, \rho_{0}\right)$, обладающий ЛСП, называется $\mathscr{L}$-гиперболическим, если

$$
\rho_{0}(x,[x, y]) \leqslant A \rho_{0}(x, y), \quad \rho_{0}(y,[x, y]) \leqslant A \rho_{0}(x, y)
$$

для некоторого $A>0$ при $\rho_{0}(x, y) \leqslant \varepsilon$ и оба отображения $f$ и $f^{-1}$ являются липшицевьми.

Целью статьи является анонс результатов о существовании ляпуновских метрик (эквивалентных исходной метрике), обладающих некоторыми оптимальными свойствами.

2. Теорема 1. Пусть гомеоморфизм $f$ обладает $Г C и \lambda_{s} \in\left[\Lambda_{s}, 1\right), \lambda_{u} \in\left[\Lambda_{u}, 1\right)$. Тогда ляпуновскую метрику $\rho_{0}$ можнно вьбрать так, что $\rho_{0}(f x, f y) / \rho_{0}(x, y) \rightarrow \lambda_{s}$ $\left(u\right.$, соответственно, $\left.\rho_{0}\left(f^{-1} x, f^{-1} y\right) / \rho_{0}(x, y) \rightarrow \lambda_{u}\right)$ равномерно по всем $x, y$ таким, что $y \in W_{\delta}^{S}(x)$ (соответственно, $\left.y \in W_{\delta}^{u}(x)\right)$ при $\rho_{0}(x, y) \rightarrow 0$ или, что равносильно, при $\rho(x, y) \rightarrow 0$.

Как отмечено в [1], обычный прием, исползуемьй для отыскания эквивалентной ляпуновской римановой метрики в случае гиперболического множества, здесь непосредственно не применим. Согласно мнению, высказанному в [1], причина этого состоит в том, что “многообразия" $W_{\delta}^{s}(x)$ и $W_{\delta}^{u}(x)$ могут не быть "гладкими" или "спрямляемыми" ни в каком, пусть даже очень слабом смысле. Однако последнее утверждение является, вообще говоря, неверным в силу следующей теоремы. 
ТеОРема 2. Пусть гомеоморфизм обладает ГС и ЛСП. Тогда ляпуновская метрика $\rho_{0}$, существование которой утверждается в теореме 1 , может быть выбрана так, что

а) для всякого $\nu>0$ найдется $r>0$ mакое, что

$$
\begin{aligned}
& \left|\rho_{0}(x,[x, y])+\rho_{0}([x, y], y)-\rho_{0}(x, y)\right| \leqslant \nu \rho_{0}(x, y), \\
& \left|\rho_{0}(x,[x, y])+\rho_{0}(x,[y, x])-\rho_{0}(x, y)\right| \leqslant \nu \rho_{0}(x, y),
\end{aligned}
$$

eсли $\rho_{0}(x, y)<r$,

б) “многообразия" $W_{\delta}^{s}(x)$ и $W_{\delta}^{u}(x)$ оказываются спрямляемыми в следующем локальном смисле: для всякого $\nu>0$ найдутся $r>0 u \tau>0$ такие, что из $y \in W_{\delta}^{\sigma}(x)$, где $\sigma=u, s, u \rho_{0}(x, y)<r, \rho_{0}(x, z)+\rho_{0}(z, y)<(1+\tau) \rho_{0}(x, y)$ вытекает $\rho_{0}\left(z, W_{\delta}^{\sigma}(x)\right)<\nu \rho_{0}(x, y)$.

Теорема 2 означает, что а) в малшіх масштабах метрика $\rho_{0}$ приблизителшно представляется как прямая сумма метрик, соответствующих каноническим координатам, и б) малые участки "многообразий" $W_{\delta}^{\sigma}(x), \sigma=u, s$ (рассмотренные при надлежащем увеличении), приблизительно являются "плоскими" подмножествами в $X$.

СледСтвиЕ (теоремы 1 и п. а) теоремы 2). Гомеоморфизм $f$ является $\mathscr{L}$-гиперболическим относительно метрики $\rho_{0}$, если были выбраны положительные $\lambda_{s} \in\left[\Lambda_{s}, 1\right)$ u $\lambda_{u} \in\left[\Lambda_{u}, 1\right)$. Более того, для всякого $\nu>0$ найдется $r>0$ такое, что если $\rho_{0}(x, y)<r$, то формульи (2) справедливы с $A=1+\nu u \rho_{0}(f x, f y) \leqslant\left(\lambda_{u}^{-1}+\nu\right) \rho_{0}(x, y)$, $\rho_{0}\left(f^{-1} x, f^{-1} y\right) \leqslant\left(\lambda_{s}^{-1}+\nu\right) \rho_{0}(x, y)$. Это означает, что в малых масштабах константа А и постояннье Липшица для отображений $f$ и $f^{-1}$ могут быть выбраны близкими $\kappa$ единице, $\lambda_{u}^{-1}$ и $\lambda_{s}^{-1}$ соответственно.

\section{СПИСОК ЛИТЕРАТУРЫ}

[1] В. М. Алексеев. Символическая динамика: Одиннадцатая матем. школа. Киев: Изд-во Ин-та математики АН УССР, 1976. [2] В. М. Алексеев, М. В. Якобсон // В кн.: Р. Боуэн. Методы символической динамики. М.: Мир, 1979. Добавление. С. 196-240. [3] М. В. Якобсон // Докл. АН CCCP. 1976. Т. 226. № 5. C. 1021-1024. [4] R. Bowen // Amer. J. Math. 1970. V. 92. №3. P. 725-747. [5] K. Sakai // Topology Appl. 1995. V. 63. №3. P. 263-266. [6] K. Sakai // Topology Appl. 2001. V. 112. № 3. P. 229-243. [7] K. Hiraide // Tokyo J. Math. 1985. V. 8. № 1. P. 219-229. [8] J. Ombach // Topology Appl. 1986. V. 23. № 1. P. 87-90.

Московский государственньй университет им. М. В. Ломоносова
Принято редколлегией 23.05 .2002 